\title{
Enhanced Holland-Batt Spline for Describing Spiral Concentrator Performance
}

\author{
J.D. Grobler, N. Naudé, J.H. Zietsman \\ Department of Materials Science and Metallurgical Engineering, University of \\ Pretoria, South Africa
}

March 11, 2016

\begin{abstract}
The Holland-Batt spline is well known when it comes to plotting spiral separation performance. The spline, which consists of a linear curve and power curve, has been successfully used to fit test work data that is presented as cumulative recovery of a valuable mineral versus the cumulative mass yield to valuable mineral concentrate. The benefit of this curve fitting process is that it produces a mathematical expression that is essential for simple mass flow modelling calculations. Although the mathematics is simple, the fitting process can be quite cumbersome. This work enhances the Holland-Batt spline with a few adjustments to improve the fit accuracy and ease the fitting process through (1) smoothing the transition zone between the linear and power law curves, (2) applying the principles to both low and high density particles, (3) use Visual Basic user-defined functions to simplify test work sheets in Excel and (4) use Excel Solver to automate the curve fitting process. These steps are applied to an example test work data set to clearly demonstrate the approach. The enhanced method is easy and simple to apply to spiral concentrator mass flow modelling.
\end{abstract}

Keywords: spiral concentrator; separation performance; spline

\section{Introduction}

The spiral concentrator, better known as a spiral, remains a competitive processing technology because it has comparatively low capital cost and high separation efficiency in a well-designed circuit configuration.

Regular assessment of spiral separation performance is important to ensure that the most suitable operational conditions (feed rate, solids concentration, medium viscosity) are applied to maintain high recoveries of valuable mineral for a specific feed material (particle size, density and shape distributions). The most common method for quantifying separation efficiency is to plot cumulative recovery of the valuable mineral versus cumulative mass yield to concentrate containing the valuable mineral. The closer the data points are to ideal recovery $(100 \%$ recovery at $100 \%$ concentrate grade), the higher the separation efficiency. This article discusses fitting of a consistent mathematical relationship of cumulative recovery versus cumulative yield to test work data points. The resulting relationship can then be used to identify data quality problems, as well as to analyse process performance. 
Linear and polynomial regression are standard methods to fit empirical models to experimental data. Test work errors can force the fitted relationship into a specific direction, which may result in a model that is not physically meaningful. There are many other equations that can be used to fit series of data points, but these may not be consistent over different test work conditions that are applied in spiral concentrator test work. Its area of applicability may be very small and will require recalibration once the operating area has shifted. This is typical of an empirical model.

The ideal equation is one that realistically describes the physical behaviour of the process under investigation, and that can be fitted accurately and consistently on test work data. This implies a model of a more fundamental nature. Such a relationship needs to be supported by large amounts of test work data and/or fundamental analysis before it can be accepted as an equation suitable to fit test work data.

The technique presented in this article is demonstrated on an example data set of a typical heavy mineral feed material with a grade of $14 \%$ by mass that was fed to a rougher spiral.

\section{Example Test Work Data}

Table 1 provides some data obtained from spiral test work. The spiral product was divided into seven mass fractions with a mouth-organ splitter. The fractions are numbered from the inside of the spiral (1) to the outside (7). The slimes content (\%SLM) of each split fraction was determined by screening on a $45 \mu \mathrm{m}$ screen. A sink-float technique with tetra-bromo ethane (TBE) at $2.98 \mathrm{~g} / \mathrm{cm}^{3}$ was used on the dried de-slimed sand fraction to determine the total heavy mineral content (\% THM, sink fraction) and remaining float fraction (\%QRT) of each split fraction. The float fraction consisted mostly of quartz. The head grade of THM, QRT and SLM were calculated with the mass weighted assay of each mass fraction. The right-hand side of Table 1 provides the cumulative figures based on the fractional values on the left-hand side.

Table 1: Example of test work data obtained from a spiral test with mouth-organ splitter. (Slimes is defined as particles smaller than $45 \mu \mathrm{m}$.)

\begin{tabular}{|c|c|c|c|c|c|c|c|c|}
\hline $\begin{array}{c}\text { Split } \\
\text { Fraction } \\
\text { No. }\end{array}$ & $\begin{array}{r}\text { Fractional } \\
\text { Mass } \\
\%\end{array}$ & $\begin{array}{r}\text { THM } \\
\text { Content } \\
\%\end{array}$ & $\begin{array}{r}\text { QRT } \\
\text { Content } \\
\%\end{array}$ & $\begin{array}{r}\text { Slimes } \\
\text { Content } \\
\%\end{array}$ & $\begin{array}{r}\text { Cum. } \\
\text { Mass } \\
\%\end{array}$ & $\begin{array}{r}\text { Cum. THM } \\
\text { Recovery } \\
\%\end{array}$ & $\begin{array}{r}\text { Cum. QRT } \\
\text { Recovery } \\
\%\end{array}$ & $\begin{array}{r}\text { Cum. Slimes } \\
\text { Recovery } \\
\%\end{array}$ \\
\hline 1 & 4.16 & 92.34 & 7.27 & 0.38 & 0.00 & 0.00 & 0.00 & 0.00 \\
\hline 2 & 3.65 & 90.91 & 8.65 & 0.44 & 4.16 & 27.95 & 0.36 & 0.60 \\
\hline 3 & 4.43 & 45.17 & 54.29 & 0.54 & 7.81 & 52.12 & 0.74 & 1.21 \\
\hline 4 & 8.27 & 13.59 & 86.18 & 0.23 & 12.25 & 66.69 & 3.62 & 2.11 \\
\hline 5 & 9.17 & 6.93 & 92.63 & 0.45 & 20.52 & 74.87 & 12.14 & 2.85 \\
\hline 6 & 34.83 & 4.81 & 93.81 & 1.38 & 29.69 & 79.49 & 22.30 & 4.40 \\
\hline 7 & 35.48 & 3.23 & 91.00 & 5.77 & 64.52 & 91.67 & 61.38 & 22.56 \\
\hline Head/Total & 100.00 & 13.74 & 83.61 & 2.64 & 100.00 & 100.00 & 100.00 & 100.00 \\
\hline
\end{tabular}

The heavy mineral material, or sink fraction, is referred to as 'high-density material' in this text; and the light minerals, or float fraction, as 'low-density material'.

\section{Holland-Batt Recovery Curve}

Holland-Batt (1990) proposed a combination of two simple equations to fit spiral recovery data. A straight line (Equation 1) and a power law (Equation 2) are combined to describe cumulative valuable mineral recovery as a function of cumulative mass yield to concentrate (Figure 11). 
In industry, this function is commonly known as a double-spline (Equation 3). Symbols are defined in the nomenclature section towards the end of this article.

$$
\begin{aligned}
r_{\text {lin }} & =a y \\
r_{\text {pow }} & =100\left(\frac{y}{100}\right)^{b} \\
r & =\min \left(r_{\text {lin }}, r_{\text {pow }}\right)=\min \left(a y, 100\left(\frac{y}{100}\right)^{b}\right)
\end{aligned}
$$

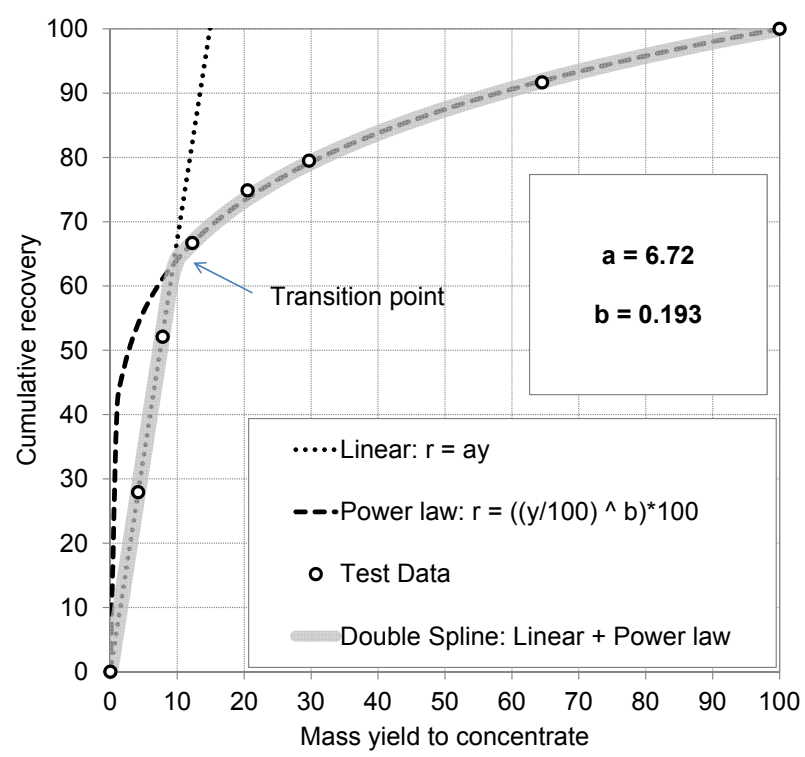

Figure 1: Holland-Batt double-spline recovery curve fitted to test-work data.

The yield-recovery curve can be divided into three zones, namely the grade zone, the transition zone and the decay zone (Figure 2a). The grade zone is primarily determined by the number of high-density particles that are concentrated at the inner side of the spiral trough, and is described by the straight line section. An increase in the number of high-density particles would result in a decrease in the gradient of the straight line, and a larger portion of the spline would be represented by the linear section.

The decay zone, described by the power law, is the result of high-density particles remaining in the bulk of low-density particles, demonstrating a steady decrease in concentration. The decay zone is influenced by the sum of all the factors that could inhibit movement of highdensity particles into the grade zone. Such factors may include increased throughput, increased solids concentration, increased viscosity (slimes content) and increased medium-density particle concentration.

Since the grade and decay zones are completely different in nature and in the separation mechanism involved, there is naturally a transition zone between them. The gradient of the curve in the transition zone would be less than that of the straight line but greater than the gradient of the power law. Holland-Batt (1990) proposed a second-order polynomial to smooth the transition from the linear section to the power law section. This adds a third section to the spline.

The Holland-Batt spline function could be fitted effortlessly to all the yield-recovery data from our test work as well as spiral testwork data from other researchers, although the secondorder polynomial caused fitting problems in some cases. The benefit of the fitted relationship is that it provides structure to the data representation within which outliers can be easily 


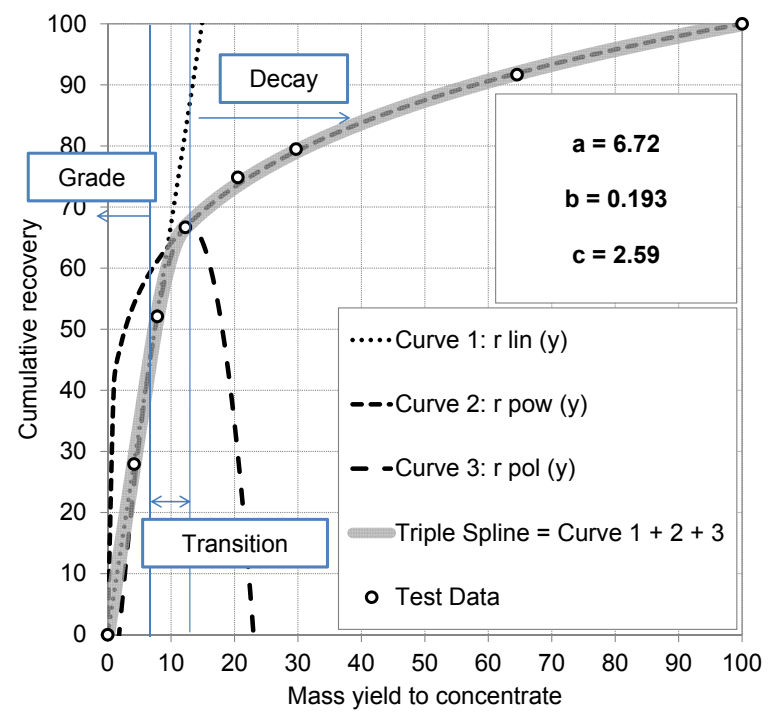

(a) Three zones covered by the triple spline.

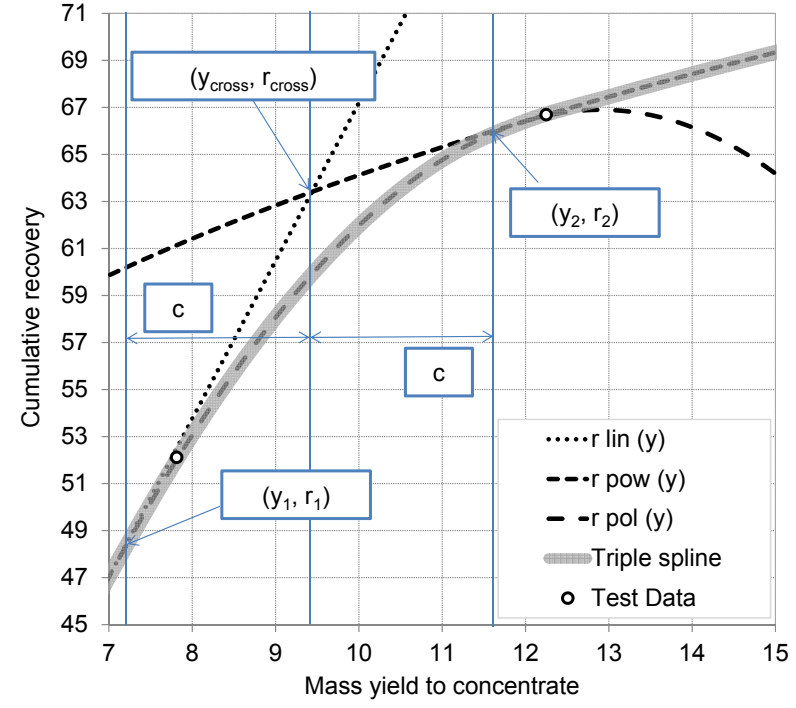

(b) Details of the transition zone.

Figure 2: Enhanced Holland-Batt triple spline for recovery of high-density material.

identified, investigated and explained. It furthermore provides a continuous relationship that can be used to calculate and plot yield-grade and yield-efficiency relationships over the entire yield range.

Figure 1 provides an example of the Holland-Batt equation (excluding the polynomial section) fitted on the yield-recovery data (cumulative THM recovery vs cumulative mass \%) of the THM assay from Table 1. The figure also shows the values for parameters $a$ and $b$ to achieve the closest fit to the testwork data.

The Holland-Batt spline function is applied to both high-density and low-density material here. For the sake of brevity, the functions are referred to as 'high-density recovery curve' and 'low-density recovery curve'.

\section{Enhanced Holland-Batt Recovery Curve}

Although the Holland-Batt spline shows reasonable agreement to test work data, the fit can often be improved, especially around the transition point from the linear section to the power law section. This is illustrated in Figure 1. Four enhancement were made to the Holland-Batt equation to improve the equation fitting process with regards to accuracy and calculation speed. These improvements are discussed in the remainder of this section.

\section{Transition Zone Polynomial}

The second-order polynomial approach used by Holland-Batt may be adequate for typical THM recovery curves, since the transition zone is small. It does however have some problems associated with it, especially for larger transitions zones that involve medium-density minerals $\left(3\right.$ to $\left.4 \mathrm{~g} / \mathrm{cm}^{3}\right)$.

The second-order polynomial uses three pieces of information to determine the three polynomial coefficients. The first is a data point located on the straight line section, and the second a data point located on the power law section. The third data point is determined somewhat arbitrarily based on the specified smoothing interval (refer to Holland-Batt 1990, p. C14). The resulting fit does not guarantee gradient continuity at the cross-over points from the linear and 
power-law sections to the polynomial section. This creates curve fitting problems in some cases.

The suggested enhancement to the Holland-Batt approach is to fit a third-order polynomial between the linear and power law sections. This function has four coefficients, and therefore requires four pieces of information to fit it. Referring to Figure 2b, the first two items are the coordinate $\left(y_{1}, r_{1}\right)$ and gradient $\left(y_{1}, r_{1}^{\prime}\right)$ at the transition from the linear section to the polynomial. The other two are the coordinate $\left(y_{2}, r_{2}\right)$ and gradient $\left(y_{2}, r_{2}^{\prime}\right)$ at the transition from the power-law to the polynomial. Once the transition zone (also called smoothing interval) halfwidth $(c)$ is specified, all four of these items become available automatically without requiring any further decisions. This method therefore does not use any arbitrary data points for the polynomial fit.

Furthermore, the fitted third-order polynomial guarantees continuity in both the recovery value and the gradient of the recovery curve. This ensures a completely smooth transition from the linear section to the power law section. The benefit of this is not clearly illustrated with the example test work data set presented here, since it has a narrow transition zone. The importance of a smooth fit does, however, become clear when spiral splitter position selections must be made within the transition zone.

This enhancement results in a triple spline that consists of a linear section $r_{l i n}(y)$, a polynomial section $r_{p o l}(y)$ and a power law section $r_{\text {pow }}(y)$. The width of the transition zone is varied to improve the accuracy of the fit.

Figure 2 demonstrates the three sections and the three parameters $(a, b$ and $c)$ used to specify the form of the triple spline. The region around the transition point in Figure 2a is enlarged in Figure $2 \mathrm{~b}$ to demonstrate the coordinates and parameters used to fit the the third-order polynomial.

Parameter $a$ specifies the gradient of $r_{l i n}(y)$, and always starts from the origin $((0,0)$ point $)$. This parameter is referred to by Holland-Batt (1990) as the 'upgrade ratio'. The higher the high-density material concentration, the lower the gradient. This parameter is constrained at the $\mathrm{y}$-axis and the gradient is rarely greater than 100, based on practical experience, and cannot be less than 1 . If the gradient is 1 , no separation occurs. If the gradient is less than one, it is described by the low-density recovery curve with its origin at the $(100,100)$ point.

Parameter $b$ specifies the power law exponent of $r_{p o w}(y)$. The higher the value of $b$, the more dilution of high-density particles into the bulk material and the lower the separation efficiency. This parameter is constrained at $b=0.001$, which is sufficiently small to match the $r=100 \%$ line, and at the zero separation line, which is at $b=1$. This recovery curve always ends in the $(100,100)$ point where $100 \%$ of the high-density particles are recovered in $100 \%$ of the mass. From a recovery point of view this parameter has the strongest influence on the high-density recovery curve.

Parameter $c$ specifies the half-width of the transition zone $\left(y_{\text {cross }}-y_{1}\right.$ or $\left.y_{2}-y_{\text {cross }}\right)$. The purpose of $r_{p o l}(y)$ is to ensure a smooth transition from $r_{l i n}(y)$ to $r_{\text {pow }}(y)$. The higher the value of this parameter, the larger is the portion of the spline that is described by $r_{\text {pol }}(y)$. The parameter is constrained between zero and $y_{\text {cross }}$ for $y$-values of less than 50. If $c$ is larger than $y_{\text {cross }}$, the spline would not include a linear section, and it would not pass through the origin. For $y$-values greater than 50,c may not exceed the value of $100-y_{\text {cross }}$ since then $r_{\text {pow }}(y)$ would be ignored and the spline would not pass through the $(100,100)$ point.

The three spline sections and their parameter limits are summarised in Table 2. The four unknown parameters $d_{0}, d_{1}, d_{2}$ and $d_{3}$ in $r_{\text {pol }}(y)$ are determined for the conditions where $r_{\text {lin }}(y)$ 
and $r_{\text {pow }}(y)$ overlap $r_{\text {pol }}(y)$ exactly. Referring to Figure $2 \mathrm{~b}$, the first overlap point $\left(y_{1}, r_{1}\right)$ is where the recovery and yield values of $r_{\text {lin }}(y)$ and $r_{\text {pol }}(y)$ are equal and the gradients are also equal:

$$
\text { At point }\left(y_{1}, r_{1}\right): \quad \begin{aligned}
r_{\text {lin }}\left(y_{1}\right) & =r_{\mathrm{pol}}\left(y_{1}\right) \\
\text { and } \quad \frac{d r_{\mathrm{lin}}\left(y_{1}\right)}{d y} & =\frac{d r_{\mathrm{pol}}\left(y_{1}\right)}{d y} \quad\left(\text { or } r_{\text {lin }}^{\prime}\left(y_{1}\right)=r_{\mathrm{pol}}^{\prime}\left(y_{1}\right)\right)
\end{aligned}
$$

Table 2: Enhanced Holland-Batt triple-spline formulae and parameter limits for high-density material recovery.

\begin{tabular}{lll}
\hline Spline Segment & Formula & Parameter Limits \\
\hline Linear & $r_{\text {lin }}(y)=a y$ & $1<a<100$ \\
Polynomial & $r_{\text {pol }}(y)=d_{3} y^{3}+d_{2} y^{2}+d_{1} y+d_{0}$ & $0<c<\left[a\left(100^{(b-1)}\right)\right]^{\frac{1}{b-1}}$ \\
& & for $0<y<50$ \\
& & $0<c<100-\left[a\left(100^{(b-1)}\right)\right]^{\frac{1}{b-1}}$ \\
& & for $50<y<100$ \\
Power Law & $r_{\text {pow }}(y)=100\left(\frac{y}{100}\right)^{b}$ & $0.001<b<1$ \\
\hline
\end{tabular}

At the second overlap point $\left(y_{2}, r_{2}\right)$ the recovery and yield values of $r_{\text {pow }}(y)$ and $r_{\text {pol }}(y)$ are equal and the gradients are also equal therefore:

$$
\text { At point }\left(y_{2}, r_{2}\right): \quad \begin{aligned}
r_{\text {pow }}\left(y_{2}\right) & =r_{\text {pol }}\left(y_{2}\right) \\
\text { and } \quad \frac{d r_{\text {pow }}\left(y_{2}\right)}{d y} & =\frac{d r_{\text {pol }}\left(y_{2}\right)}{d y} \quad\left(\text { or } r_{\text {pow }}^{\prime}\left(y_{2}\right)=r_{\text {pol }}^{\prime}\left(y_{2}\right)\right)
\end{aligned}
$$

These conditions result in four unknowns $\left(d_{0}, d_{1}, d_{2}\right.$ and $\left.d_{3}\right)$ and the following four equations:

$$
\begin{gathered}
r\left(y_{1}\right)=d_{3} y_{1}^{3}+d_{2} y_{1}^{2}+d_{1} y_{1}+d_{0}=a y_{1} \\
r^{\prime}\left(y_{1}\right)=3 d_{3} y_{1}^{2}+2 d_{2} y_{1}+d_{1}=a \\
r\left(y_{2}\right)=d_{3} y_{2}^{3}+d_{2} y_{2}^{2}+d_{1} y_{2}+d_{0}=100\left(\frac{y_{2}}{100}\right)^{b} \\
r^{\prime}\left(y_{2}\right)=3 d_{3} y_{2}^{2}+2 d_{2} y_{2}+d_{1}=100^{(1-b)} b y_{2}^{(b-1)}
\end{gathered}
$$

This is a system of linear equations in $d_{i}$ that can be solved with matrix algebra. Equations 4 and 5 express the equations in matrix notation.

$$
\begin{aligned}
{\left[\begin{array}{cccc}
y_{1}^{3} & y_{1}^{2} & y_{1} & 1 \\
3 y_{1}^{2} & 2 y_{1} & 1 & 0 \\
y_{2}^{3} & y_{2}^{2} & y_{2} & 1 \\
3 y_{2}^{2} & 2 y_{2} & 1 & 0
\end{array}\right]\left[\begin{array}{l}
d_{3} \\
d_{2} \\
d_{1} \\
d_{0}
\end{array}\right] } & =\left[\begin{array}{c}
a y_{1} \\
a \\
100\left(\frac{y_{2}}{100}\right)^{b} \\
100^{(1-b)} b y_{2}^{(b-1)}
\end{array}\right] \\
\mathbf{Y d} & =\mathbf{r}
\end{aligned}
$$

The values of the unknowns are determined by Equation 6 .

$$
\mathbf{d}=\mathbf{Y}^{-1} \mathbf{r}
$$

Combining the formulas of the three sections, Equation 7 describes the high-density recovery triple spline.

$$
r(y)= \begin{cases}r_{\text {lin }}(y)=a y, & \text { if } 0 \leq y<\left(y_{\text {cross }}-c\right) \\ r_{\text {pol }}(y)=d_{3} y^{3}+d_{2} y^{2}+d_{1} y+d_{0}, & \text { if }\left(y_{\text {cross }}-c\right) \leq y \leq\left(y_{\text {cross }}+c\right) \\ r_{\text {pow }}(y)=100\left(\frac{y}{100}\right)^{b}, & \text { if }\left(y_{\text {cross }}+c\right)<y \leq 100\end{cases}
$$




\section{User-defined Functions}

An Excel Visual Basic module was created to implement Equations 7 and 10 as user-defined functions (named Spline_HighDensity and Spline_LowDensity). The functions require the user to supply four parameters $(a, b, c$ and $y)$ and calculates cumulative recovery. This avoids having to do the same calculations many times over inside spreadsheet cells, which is time consuming and error prone. All the calculations are therefore done in the background, which makes it user friendly and keeps the data sheets dealing with large volumes of data neat and clear. The Visual Basic code for the two functions are provided in Listings 1 and 2.

Limiting conditions for the permissible envelope of separation are also considered and programmed to ensure that the functions remain robust over the entire range of input parameter value. An error message guides the user if spline parameter values are specified that violate the permissible separation envelope.

This envelope is limited on the right side by the zero separation line that intersects the origin and the $(100,100)$ point, on the left side by the theoretical grade line $\left(r=\frac{100 y}{\text { grade }}\right)$, and by the $r=100$ horizontal line on top. Figure 3 shows this envelope.

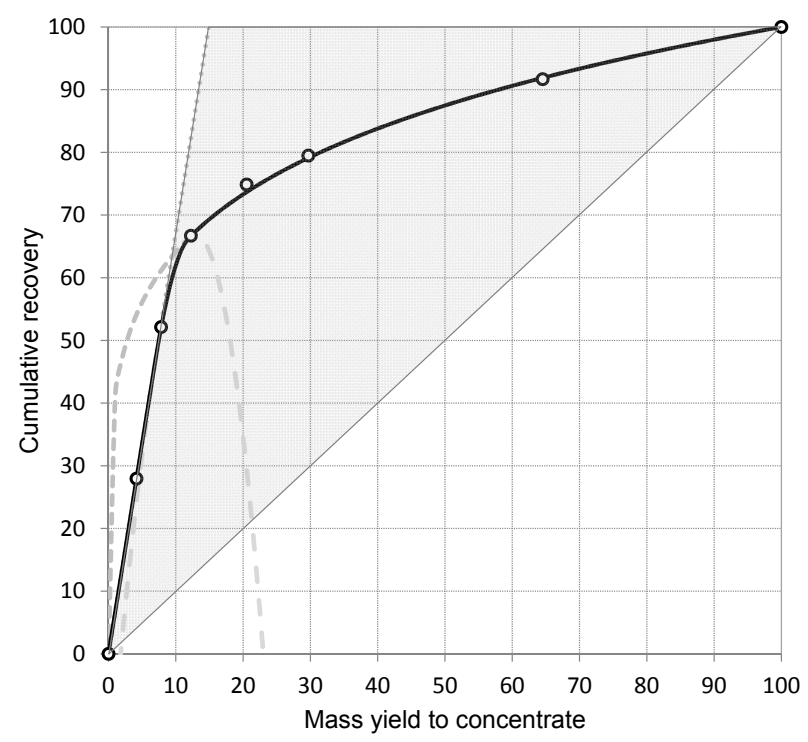

Figure 3: Permissible envelope for the high-density recovery curve (indicated in grey).

\section{Automated Curve Fitting}

Selecting the three parameters $(a, b$ and $c)$ to arrive the best fit to a test work data set may be counter intuitive to the user and it may take a long time. Different users can also have different parameter selection results for the same test work data set. To avoid these problems the procedure of fitting a spline to test work data was automated.

A macro was created in a second Excel Visual Basic module to rapidly calculate the spline parameters $(a, b$ and $c$ ) to produce the fit that minimises the error calculated with Equation 8. The macro code is not provided here, since it is specific to the layout of the worksheets we used. The macro inputs are:

- Starting values for $(a, b$ and $c)$.

These are determined through visual evaluation by plotting the recovery spline and testwork data points on the same graph, similar to Figure 1. As long as reasonable values are provided, the optimisation routine is not very sensitive to the starting values. 
- The test work data set.

The cumulative mass and cumulative THM recovery columns of Table 1 is an example of what the macro would use. It consists of seven separate cuts in the example data set.

Determining the optimal set of spline parameters is a non-linear constrained optimisation problem. The macro uses Excel's Solver facility to find a solution. Equation 8 is the objective function. The $r_{\mathrm{i}}$ (spline) terms are calculated with the user-defined functions described in the previous section. Parameters $a, b$ and $c$ are the optimisation variables adjusted to minimise the objective function. The values of these variables are constrained according to the parameter limits in Table 2 and Table 3 to force the spline to remain inside the valid separation envelope. This curve fitting process takes only a few seconds and many data fits can be done in a short period of time.

$$
\text { sum of squared errors }=\sum_{i=1}^{7}\left(r_{\mathrm{i}(\text { test work })}-r_{\mathrm{i}(\text { spline })}\right)^{2}
$$

\section{Low-density Recovery Curve}

In almost all relevant literature references (Henderson and MacHunter 2003; Richards and Palmer 1997; Holland-Batt 1995) only the recovery of high-density material (THM) is plotted. The recovery (or rejection) of low-density material (QRT) is omitted and as a result not fitted. The position and consistency of the recovery curve for low-density material can be crucial for spiral performance assessment since it usually represents more than $80 \%$ of the mass on the spiral and a small movement in this curve can result in a significant influence on mass yield. The Holland-Batt equation was further extended to accommodate the recovery of low-density material.

For the power law to function correctly, it was mirrored to the $(100,100)$ point as the new origin. The high-density formulae and parameter limits in Table 2 and Equation 7 are converted to low-density equivalents by replacing $(y)$ with $\left(y^{*}\right)$, and $(r)$ with $\left(r^{*}\right)$. The mirror calculations are shown in Equation 9. The resulting low-density equivalents are shown in Table 3 and Equation 10.

$$
\text { Mirror } \begin{cases}\text { Yield } & y^{*}=100-y, \\ \text { Recovery } & r^{*}=100-r\end{cases}
$$

Table 3: Enhanced Holland-Batt triple-spline formulae and parameter limits for low-density material recovery.

\begin{tabular}{lll}
\hline Spline Segment & Formula & Parameter Limits \\
\hline Linear & $r_{\text {lin }}^{*}\left(y^{*}\right)=a y^{*}$ & $1<a<100$ \\
Polynomial & $r_{\text {pol }}^{*}\left(y^{*}\right)=d_{3}\left(y^{*}\right)^{3}+d_{2}\left(y^{*}\right)^{2}+d_{1}\left(y^{*}\right)+d_{0}$ & $0<c<\left[a\left(100^{(b-1)}\right)\right]^{\frac{1}{b-1}}$ \\
& & for $0<y^{*}<50$ \\
& & $0<c<100-\left[a\left(100^{(b-1)}\right)\right]^{\frac{1}{b-1}}$ \\
& & for $50<y^{*}<100$ \\
Power Law & $r_{\text {pow }}^{*}\left(y^{*}\right)=100\left(\frac{y^{*}}{100}\right)^{b}$ & $0.001<b<1$ \\
\hline
\end{tabular}

$$
r(y)= \begin{cases}r_{\text {lin }}^{*}\left(y^{*}\right)=a y^{*}, & \text { if } 0 \leq y^{*}<\left(y_{\text {cross }}^{*}-c\right) \\ r_{\text {pol }}^{*}\left(y^{*}\right)=d_{3}\left(y^{*}\right)^{3}+d_{2}\left(y^{*}\right)^{2}+d_{1}\left(y^{*}\right)+d_{0}, & \text { if }\left(y_{\text {cross }}^{*}-c\right) \leq y^{*} \leq\left(y_{\text {cross }}^{*}+c\right) \\ r_{\text {pow }}^{*}\left(y^{*}\right)=100\left(\frac{y^{*}}{100}\right)^{b}, & \text { if }\left(y_{\text {cross }}^{*}+c\right)<y^{*} \leq 100\end{cases}
$$


As expected, the low-density recovery curve is dominated by the grade zone, which is located on the outside of the spiral. Figure $4 a$ demonstrates the different spline sections for lowdensity material recovery and Figure $4 \mathrm{~b}$ provides detail of the transition zone. The permissible separation envelope for the recovery of low-density material is also significantly smaller (Figure 5), since it represents the bulk of the mass on the spiral.

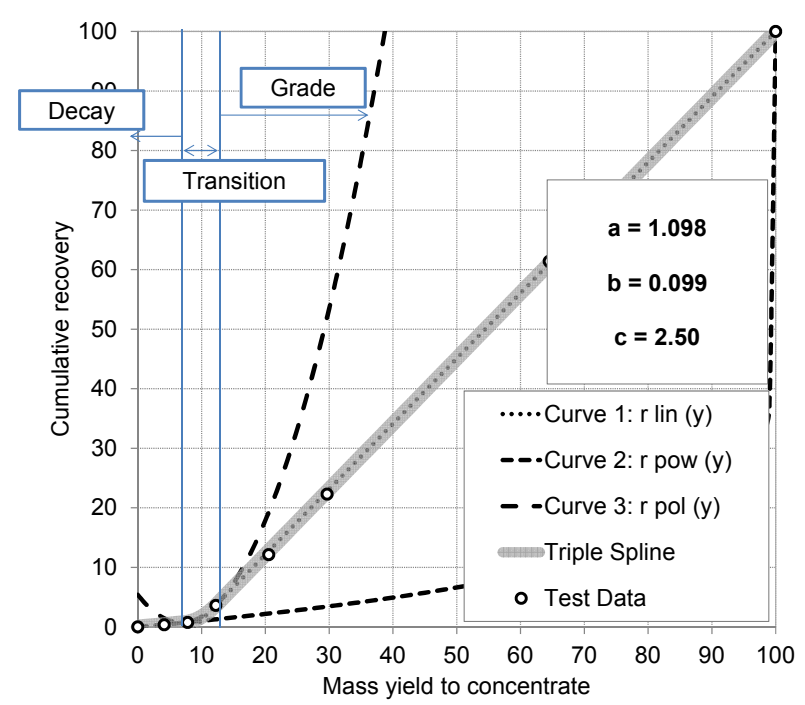

(a) Three zones covered by the triple spline.

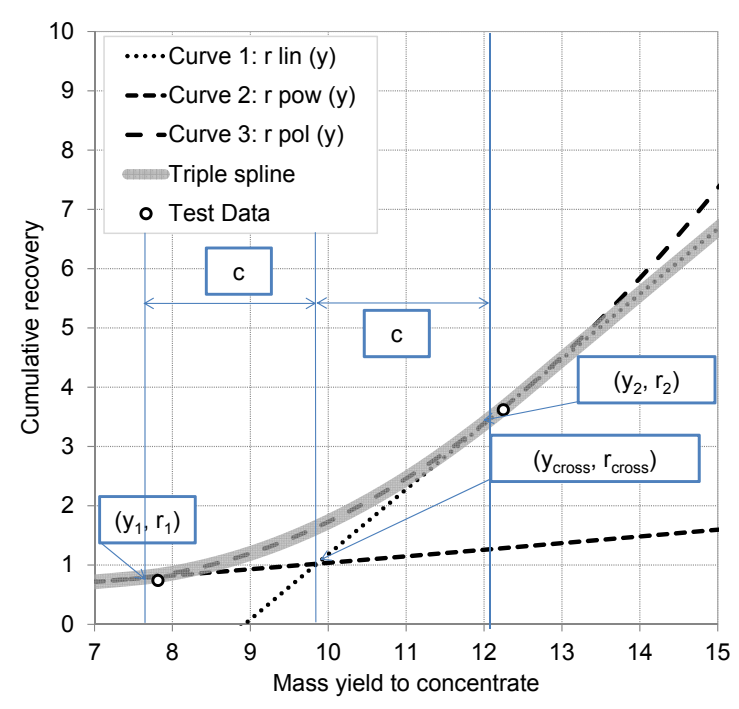

(b) Details of the transition zone.

Figure 4: Enhanced Holland-Batt triple spline for low-density material recovery.



Figure 5: Permissible envelope for the low-density material recovery curve (indicated in grey).

\section{Conclusions}

This simple, yet effective curve fitting method can be applied to all spiral test-work data to create a robust mathematical relationship for recovery versus yield, which can be used easily for mass balance calculations on spiral circuits. Spiral separation efficiency can also be measured more consistently. 
The mathematical equations used are simple, with only three parameters. These triple spline parameters can also be correlated well to spiral separation behaviour (grade, decay and transition), which makes them physically meaningful.

The enhancements made to the Holland-Batt recovery curve in this study have important benefits, such as performing a mass balance of multiple density classes (high, medium and low). The automated fitting assists in dealing with large data sets and it also improves the accuracy and speed of the curve fitting process. The user-defined functions keep the test-work sheets in Excel neat and user friendly.

\section{Nomenclature}

\section{Symbols}

a gradient of straight line segment, dimensionless

$b$ exponent of power law segment, dimensionless

$c$ transition zone half width, mass $\%$

$d_{j}$ coefficient for polynomial order $j$ term

$r$ cumulative high-density material recovery, mass $\%$

$r^{*} \quad$ cumulative low-density material recovery, mass $\%$

$y$ cumulative mass yield to high-density concentrate, mass $\%$

$y^{*} \quad$ cumulative mass yield to low-density tailings, mass $\%$

\section{Subscripts}

cross the point where the linear and power-law sections cross

i spiral split fraction index

lin linear section

pol polynomial section

pow power-law section

\section{Acknowledgements}

The authors thank Exxaro Resources for permission to publish this work.

\section{References}

Henderson, D. and D. MacHunter (2003). A review of spiral technology for fine gravity beneficiation. Internal report. MD Mineral Technologies.

Holland-Batt, D. (1990). "Interpretation of spiral and sluice tests". In: Transactions Institution of Mining and Metallurgy 99, pp. 11-20.

- (1995). "Some design considerations for spiral separators". In: Mineral Technologies, pp. 116.

Richards, R. and M. Palmer (1997). "High capacity gravity separators - a review of current status". In: Minerals Engineering 10, pp. 973-982.

\section{Code Listings}

Listing 1: Visual Basic code for calculating high-density material recovery curve.

1 Public Function Spline_HighDensity (a As Double, b As Double, c As Double, y As Double) 


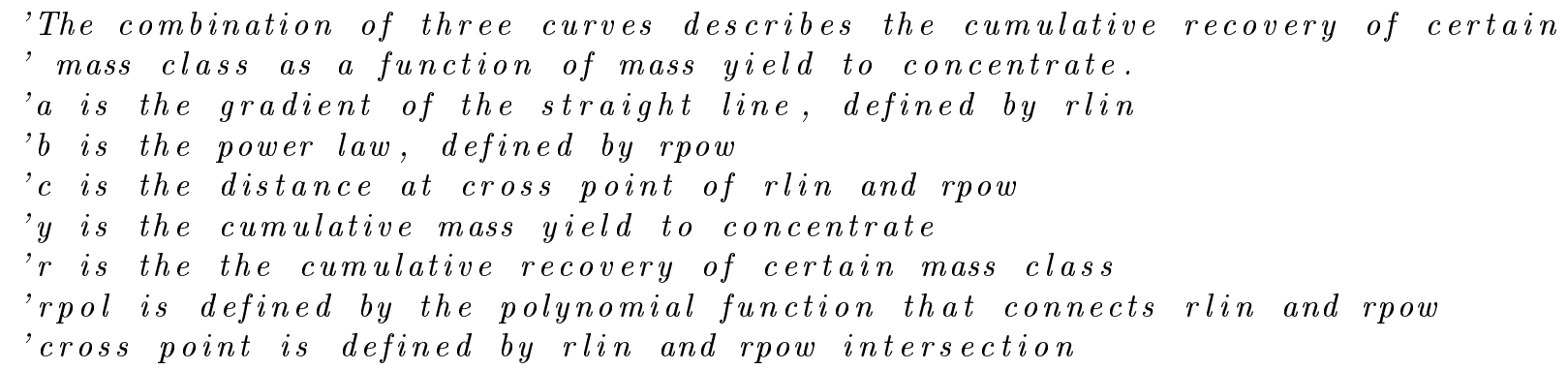

Dim rlin As Double, rpow As Double

$\mathrm{rlin}=\mathrm{a} * \mathrm{y}$

rpow $=100 *((y / 100) \wedge b)$

Dim y_cross As Double

$\mathrm{y}_{-} \operatorname{cross}=(\mathrm{a} *(100 \wedge(\mathrm{b}-1))) \wedge(1 /(\mathrm{b}-1))$

Dim y1 As Double, y2 As Double, r1 As Double, r 2 As Double

Dim r1_ As Double, r2 _ As Double

$\mathrm{y} 1=\mathrm{y}_{-} \mathrm{cross}-\mathrm{c}$

$\mathrm{y} 2=\mathrm{y}_{-} \operatorname{cross}+\mathrm{c}$

$\mathrm{r} 1=\mathrm{a} * \mathrm{y} 1$

$\mathrm{r} 2=100 *((\mathrm{y} 2 / 100) \wedge \mathrm{b})$

$\mathrm{r} 1_{-}=\mathrm{a}$

$\mathrm{r}_{-}^{-}=\left(\left(100 *\left(\left(\left(\mathrm{y}_{-} \operatorname{cross}+\mathrm{c}+1\right) / 100\right) \wedge \mathrm{b}\right)-\left(100 *\left(\left(\left(\mathrm{y}_{-} \operatorname{cross}+\mathrm{c}\right) / 100\right) \wedge \mathrm{b}\right)\right)\right)\right)-$ $/\left(\left(y_{-} \operatorname{cross}+\mathrm{c}+1\right)-\left(\mathrm{y}_{-} \operatorname{cross}+\mathrm{c}\right)\right)$

Dim Z As Variant

ReDim Z $(4,4)$ As Double

$\mathrm{Z}(1,1)=(1)$

$\mathrm{Z}(1,2)=(\mathrm{y} 1)$

$\mathrm{Z}(1,3)=(\mathrm{y} 1 \wedge 2)$

$\mathrm{Z}(1,4)=(\mathrm{y} 1 \wedge 3)$

$\mathrm{Z}(2,1)=(1)$

$\mathrm{Z}(2,2)=(\mathrm{y} 2)$

$\mathrm{Z}(2,3)=(\mathrm{y} 2 \wedge 2)$

$\mathrm{Z}(2,4)=(\mathrm{y} 2 \wedge 3)$

$\mathrm{Z}(3,1)=(0)$

$\mathrm{Z}(3,2)=(1)$

$42 \mathrm{Z}(3,3)=(2 * \mathrm{y} 1)$

$43 \mathrm{Z}(3,4)=(3 * \mathrm{y} 1$ ^ 2$)$

$44 \mathrm{Z}(4,1)=(0)$

$45 \mathrm{Z}(4,2)=(1)$

$46 \mathrm{Z}(4,3)=(2 * \mathrm{y} 2)$

$47 \mathrm{Z}(4,4)=(3 * \mathrm{y} 2 \wedge 2)$

48

49 Dim R As Variant

$50 \operatorname{ReDim} \mathrm{R}(4,1)$ As Double

$51 \mathrm{R}(1,1)=(\mathrm{r} 1)$

$52 \mathrm{R}(2,1)=(\mathrm{r} 2)$

$53 \mathrm{R}(3,1)=\left(\mathrm{r} 1_{-}\right)$

$54 \mathrm{R}(4,1)=\left(\mathrm{r}_{-}^{-}\right)$

Dim Zinverse As Variant

Zinverse $=$ WorksheetFunction. MInverse $(Z)$

Dim Coeff As Variant

Coeff $=$ WorksheetFunction.MMult(Zinverse, R) 
Dim d0 As Double, d1 As Double, d2 As Double, d3 As Double

$\mathrm{d} 0=\operatorname{Coeff}(1,1)$

$\mathrm{d} 1=\operatorname{Coeff}(2,1)$

$\mathrm{d} 2=\operatorname{Coeff}(3,1)$

$\mathrm{d} 3=\operatorname{Coeff}(4,1)$

Dim rpol As Double

$\mathrm{rpol}=\mathrm{d} 0+\mathrm{d} 1 * \mathrm{y}+\mathrm{d} 2 * \mathrm{y} \wedge 2+\mathrm{d} 3 * \mathrm{y} \wedge 3$

If $\mathrm{y}<=\mathrm{y} 1$ And $\mathrm{y}>=0$ Then

Spline_HighDensity $=$ rlin

Else If $\mathrm{y}<=\mathrm{y} 2$ And $\mathrm{y}>\mathrm{y} 1$ Then

Spline_HighDensity $=$ rpol

Else If $\mathrm{y}>\mathrm{y} 2$ And $\mathrm{y}<=100$ Then

Else

Spline_HighDensity $=$ rpow

End If

Spline_HighDensity = "unknown"

81 End Function

Listing 2: Visual Basic code for calculating low-density material recovery curve.

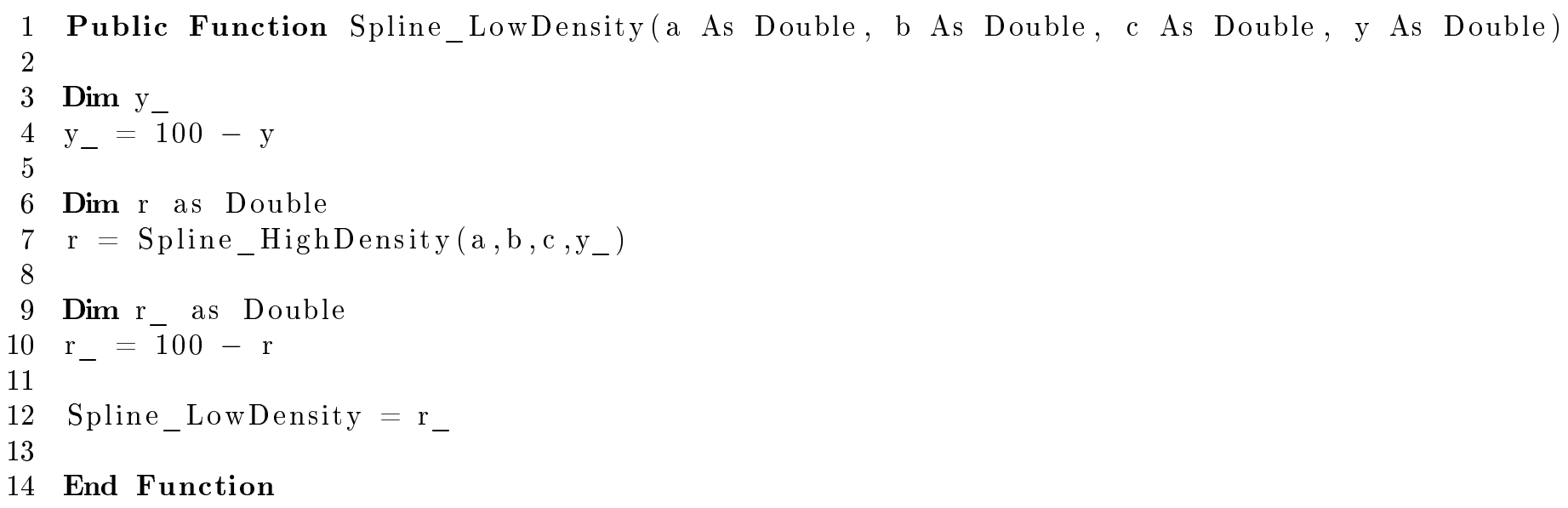

